die Grenzen der Provinz dringt nur wenig Kenntnis von ihrem Wesen und Wert. Jenseits Berlins, je weiter man zumal nach Westen und Süden dringt, ist der alte Bann noch nicht gebrochen, auch den preußischen Anteil 'des ehemaligen Königreiches Polen als ein zweites Sibirien anzusehen, ungastlich, unwirtlich, ohne Kultur. Jetzt blickt die Welt hierher - das alte Königtum, freilich nicht in früherem Umfange, soll erstehen. Unleugbar gibt es eine hochstehende polnische Kultur, welche nicht in Museen begraben ist, sondern lebt.

Wandel der Zeiten! In einem alten Buche, welches der Feder eines polnischen Arztes, des Matthias aus Miechow, entstammt - er starb 1523 zu Krakau - und das die Geschichte Polens behandelt, lesen wir: „In diesem Lande pflügt man nicht und sät man nicht. Brot und Geld gibt es nicht. Man lebt von dem reichlichen Wilde der Wälder. Vernunftlos wie Bestien kleidet man sich mit Fellen, von denen die, verschiedenartigsten aneinander genäht werden, vom Wolf, Hirsch usw., wie der Zufall sie gerade bringt."

Geschmückt mit der Würde eines Magisters war dieser Matthias Miechovius in die Welt gezogen, Wortkämpfe zu führen an allen Universitäten Italiens und Deutschlands. Armselig sah es ja alle Zeit in den medizinischen Fakultäten der Akademien Polens aus. Da trieb es die Jugend fort in die Welt, bis die Tüchtigsten ihr höchstes.Glück erreichte, ein Ruf als Leibarzt eines Bischofs, Fürsten oder gar des Königs. So wurde auch Matthias Leibarzt des Königs von Polen, Sigismund I. Aber bald hielt es ihn nicht mehr in der Hofluft. Thn erfaßte der Drang, seinem Vaterland mehr zu sein. In außerordentlicher Vielseitigkeit, nachdem er die kirchlichen Weihen genommen, widmete er sich als Lehrer der Astrologie und Medizin an den privaten und klösterlichen Schulen seiner Heimat Miechow dem Unterricht der Jugend. Er hinterließ uns ein Büchlein: De conservanda sanitate.

Um die Bedeutung des hervorragendsten der polnischen Aerzte, des Josef Struthius, richtig zu erkennen, ist es erforderlich, mit wenigen Strichen die medizin-historischen Grundzüge seiner Zeit zu skizzieren.

Der Einfluß, welchen Hippokrates auf das wissenschaftliche Denken der Aerzte ausgeübt hatte, war längst der rein praktischen Medizin Galen s gewichen. Aber schon im 15. Jahrhundert machte sich selbständiges Beobachten, Denken und Handeln im Bereiche der Naturwissenschaft und Heilkunde bemerkbar. Der eigentliche Wiedererwecker dieser Richtung war ein Deutscher: Theophrastus Paracelsus von Hohenheim, der deutsche Hi p pokrates. Die in jene Zeit fallenden Seuchen, vor allem die Syphilis und der Petechialtyphus, gaben Beobachtungsstoff in Menge. "Anderen galt es wieder, im Kampfe gegen Galen, den alten, ursprünglichen Hippokrates in Schrift und Praxis lebendig zu machen. Da blühten die Kämpfe um Streitfragen noch das ganze 16. Jahrhundert hindurch - Aderlaß, Harnschau, Pulslehre schwanden als wissenschaftliche Themen nicht von der Tagesordnung. Der Puls sollte über alles Aufschlu $ß$ geben, was mit den Lebenskräften zusammenhängt. Während dieser für Hi p po krates nur im Zusammenhang mit anderen Erscheinungen am Körper zur Erkennung von Krankheiten diente, genügte für eine große Anzahl der damaligen Aerzte - Spezialisten auf ihrem Gebiete - schon die bloße Harnschau oder das Fühlen des Pulses zur Erkennung der Veränderungen im Körper - pulsus bonus, urina bona et aeger moritur, so lau tet ein Spottwort jener Zeit. Auf diesem Boden entstand Josephi Struthii ,Ars sphygmographica, seu pulsum doctrina, supra MCC annos perdita et desiderata, omnibus tamen medicinam cum nominis celebritate maximaque utilitate facere volentibus summe necessaria, Libris V. Basi. 1540.“ Er stellte 15 einfache und 17 zusammengesetzte Arten des Pulses auf und erklärte die Rhythmen des Pulses nach musikalischen Gesetzen. Dabei ging er ausführlich auf die Ursachen der verschiedenartigen Veränderungen ein. Das Buch, welches noch heute seines medizin-historischen und musikwissenschaftlichen Bearbeiters harrt, erntete so vielen Beifall, daß an einem einzigen Tage 800 Exemplare davon verkauft wurden. Diesem Erfolge verdankte der Sohn der Stadt Posen, nachdem er jahrelang in Padua Medizin gelehrt hatte, seinen schließlichen Ruf als Leibarzt des Königs Sigismund August von Polen, an dessen Hofe er im Jahre $1568 \mathrm{im}$ Alter von 58 Jahren starb. Von Strus - so sein Name in polnischer Version - stammt die erste lateinische Uebersetzung von fünf Galenischen Büchern. Und diesem Studium verdankte er die Anregung zu dem überaus mühseligen Werke über die längst vergessene Pulslehre. In der Chronik Posens wird er als ein zwar tüchtiger, aber sehr habsüchtiger Arzt dargestellt. Er starb als reicher Mann. Seine Gebeine ruhen im Posener Dome, wo ein Grabmal ihn als

\title{
Geschichte der Medizin.
}

\section{Polen in der Geschichte der Medizin.}

\section{Von Dr. Carl Kassel in Posen.}

Nur einige Stunden Fahrt im bequemen D.Zuge trennt die Hauptstadt der Ostmark von der Kapitale des Deutschen Reiches. Aber über

1) L. Traube, Gesammelte Beiträge zur Pathologie u. Physiologie 2 S. 664. - 2) Jahrg. 1864 Nr. 2.
Artis Sphygmicae per tot saecula oblitae, novas restaurator preist.

In Posen lebte ferner im 16. Jahrhundert der aus Masovien stam. mende Stanislaus Chrościewski, mit dem Beinamen Niger. Er hatte in Leipzig studiert und noch hier sein ,Epicedium in mortem im. maturam illustrissimi principis domini Joannis de ducibus Lithuaniae Praesulis posnanensis (1538) ${ }^{c}$. herausgegeben. Von ihm stammen außerdem eine Elegie auf den Wert der Karlsbader Heilquelle und zwei Arbeiten „De humoribus" und „De odore“. Bei seinem Könige Sigismund August stand er in großem Ansehen. Er war 1567 Bürgermeister von Posen. 
Noch ein anderer Leibarzt von Sigismund August verdient erwähnt zu werden, Adalbert aus Posen. In seinem Buche „De Bona militum valetudine" schreibt Sneberger: „Sed alii de rerum a me expositarum dignitate ac utilitate, imprimis vero doctissimi S. R. M. tuae medici honoris causa hic a me nominandi, Albertus et Petrus Posnaniensis etc."

Die Vollständigkeit dieser Skizze erfordert noch die Erwähnung einiger anderer Aerzte Polens. Ein Schüler der Universität Krakau, Ausländer zwar, aus Pilsen gebürtig, war Sebastianus Patricius. Im. Jahre 1583 erwarb er sich die Magisterwürde, reiste dann nach Belgien und Italien, wurde in Padua mit dem Doktorgrade beschenkt. Später bekleidete er in Polen mehrere Leibarztstellen und starb 1610. Er schrieb ein Buch „Remedium contra pestilentiae vim".

Ein weiterer Ausländer, Antoni us For mica, geboren zu Messina, spielt in der Geschichte der Aerzte Polens eine gewisse Rolle. Nach Beendigung seines medizinischen Studiums widmete er sich auf Grund eines während schwerer Krankheit abgelegten Gelübdes der Theologie und war mit päpstlicher Erlaubnis als Priester und Arzt tätig. In späteren Jahren ging er als Leibarzt des Fürstbischofs nach Krakau. Auf dessen Befehl gab er im Frühjahr 1737 ein „Consilium Medicum, pro avertenda et curanda Lue Epidemica, in Tractu Varsaviensi, anno eodem 1737 grassante" zu Warschau heraus.

Man blättert vergebens in den Annalen der polnischen Geschichte -eine reiche Ausbeute beim Suchen nach bedeutenden polnischen Aerzten in alter Zeit gibt es nicht. Als das Polenreich sich dem Untergang näherte, zeigten die polnischen Universitäten am frühesten die Zeichen des Verfalls. Die Besetzung der medizinischen Fakultät Krakaus bestand aus fünf Lehrkräften, je einem Anatomen, Physiologen, Pathologen, Botaniker und Therapeuten. Die medizinische Fakultät Zamoisz hatte schon am Ende des 17. Jahrhunderts aufgehört zu sein!

Im Anfange des 16. Jahrhunderts begegnen wir in Polen den ersten jüdischen Aerzten. Sie und ihre Nachfolger bilden ein für die Kulturgeschichte Polens überaus wichtiges eigenes Kapitel. Ihre ärztliche Bildung holten sie sich im Auslande, da ihnen die Universität Krakau verschlossen war. Daß die tüchtigen, gewissenhaften, fleißigen, nüchternen jüdischen Aerzte, je mehr sich ihrer in Polen niederließen, eine störende Konkurrenz wurden, ist nicht weiter zu verwundern- Ebensowenig, da $B$ die damalige Richtung ärztlicher Standesehre bei der Kommission der Guten Ordnung im Jahre 1779 die Bestimmung durchsetzte: „Kein jüdischer Arzt soll sich unterstehen, einen Christen zu behandeln". Schon Wladislaus IV. (1632-1648) hatte übrigens einen gleichen Befehl erlassen.

Es ist dem Plauderer schwer, beim Rückblick auf die ärztlichen Verhältnisse Großpolens Maß zu halten. Bietet sich doch schier unerschöpflicher Stoff. Streng Halt aber möchte ich an der Stelle machen, da die Interessen der Medici, Chirurgi, Tonsores, Balneatores sich scheiden, ohne freilich jenes köstliche Klagelied verschweigen zu können, welches im Jahre 1715 entstand und gegen die jüdische Konkurrenz gericlitet war:

$$
\text { Chirurgi. }
$$

Plorat, chyrurgus: - ille Licurgus

Lege non valet, - Judaeus mallet

Cunctis mederi. - Sciant posteri.

Is cicatrices, - Pedes, pollices,

Curat medellis - viris, faemellis,

Tundit et crines - spectantur fines.

Tonsor libitu - suo habitu

Tonsor ubivis - quaerito, si vis,

Sunt nostri tonsi - sponsae et sponsi.

Die ärztliche Versorgung Polens war überaus schlecht. Fs gab zur Zeit des Verfalls nur wenige promovierte, noch weniger examinierte und approbierte Aerzte. Es genügte, der Sohn eines Doktors zu sein, um ein Testimonium capacitatis zur Ausübung innerer Kuren zu erhalten. Im übrigen mußten die damals nicht gerade auf stolzer Höhe stehenden Soldatenärzte einspringen. Als die preußische Regierung ihr Teilungsgebiet übernahm, machte sie vergebliche Anstrengungen, deutsche Aerzte gegen schlechte Bezahlung in Polen anzusiedeln. Aehnlich wie heute lautete der damalige amtliche Bericht: „Da nur wenige von ihnen der polnischen Sprache mächtig sind, oder sich entweder mit der lateinischen oder einem Translateur forthelfen müssen, so ist ihre Praxis höchst unbedeutend." In einem anderen Berichte von damals lesen wir über die promovierten Aerzte: „.. . . . einesteils waren sie in sich selbst mangelhaft, denn diese sogenannten promovierten Aerzte waren regelmäßig unwissende und jeder Korruption empfängliche Abenteuerer aus dem Auslande ......" Ein Bild — kurz aber klar.

Gleichwohl ließ es die polnische Regierung nicht an Versuchen fehlen, für Polen tüchtige Aerzte heranzubilden. Im Jahre 1784 gab König Stanislaus August dem Vorschlage der Erziehungskommission Gesetzeskraft, wonach aus jedem der königlichen Güter im ganzen Lande ein junger Menzch zur Erlernung der Arzneiwissenschaft und der Medizin auf die Universität Krakau oder Wilna geschickt werden sollte. Sofort zogen einige Hundert junger Leute nach Krakau. Die Lücken in ihrer für das akademische Studium notwendigen Vorbildung sollten durch die Teilnahme an einer Art Vorbereitungsunterricht ausgefüllt werden. Die jüngsten Zöglinge mußten in niedrige Schulen gehen, die tauglicheren hörten Physik, Anatomie, Physiologie usw. Die Ausbildung wurde durch eine Prüfung abgeschlossen, nach deren Bestehen die Kandidaten in ihre Heimat zurückkehren sollten, um dort die Stellen von Kreisphysikern und Chirurgen zu übernehmen.

Als nun Preußen die Herrschaft in dem ihm zugefallenen Teile Polens antrat, traf die Regierung die Bestimmung, daß von nun an jedes polnische Departement halbjährig einen Zögling in die Berliner Pepinière schicken sollte, die Bildungsanstalt Berlins, welche 1795 zur Heranbildung tüchtiger Feldärzte gegründet worden war und deren Schüler sich vorerst meist aus den Kreisen der Barbiergehilfen rekrutierten.

Ein Schritt weiter in dieser Betrachtung würde gar zu leicht in das Gebiet der Gegenwartspolitik führen. Die Arztfrage der Ostmark ist ein wichtiger Teil der Ostmarkenfrage: denn der Arzt wie jeder Akademiker überhaupt übernimmt hier die Rolle des nationalpolitischen Kulturträgers und Kämpfers. Die preußische Regierung hat hiernach ihre Maßnahmen getroffen. $\mathrm{Ob}$ sie die richtigen waren, das zu beurteilen, ist hier nicht der Ort. Eines aber dürfen wir ohne Scheu aussprechen: Der polnische Arzt von heute ist das gerade Gegenteil von seinem Vorgänger in dem ehemaligen Polen. Er ist ein geistig und sittlich hochstehender Kulturfaktor, welcher an allen denjenigen Vorteilen kultureller Erziehung auf das eifrigste teilnimmt, welche die neue Herrschaft ihm bietet. 